\title{
Procesos de interacción comunicacional de niños y niñas cuya madre está privada de la libertad en el Centro de Rehabilitación Social Femenino de Quito
}

Communicational interaction processes of children whose mother is imprisoned in the Women's jail of Quito

Margarita Estefanía Cortes Morejón. ${ }^{1}$, Nathalie Geoconda Ortiz López. ${ }^{2}$ \& Lourdes

Yessenia Cabrera Martínez. ${ }^{3}$

Recibido: 11-07-2020 / Revisado: 13-08-2020 / Aceptado: 14-09-2020 / Publicado: 03-10-2020

\begin{abstract}
Resumen.
DOI: https://doi.org/10.33262/concienciadigital.v3i4.1429

La problemática vinculada a infancia y prisión es una temática escasamente analizada desde el campo de la comunicación, por ello el objetivo del presente artículo es exponer los procesos de interacción comunicacional que se dan entre madres e hijos que permanecen al interior del Centro de Rehabilitación Social (CRS) Femenino de Quito, Ecuador. La estrategia metodológica se basó en un diseño no experimental, de carácter transversal, a través de un enfoque cualitativo mediante la técnica de entrevistas en profundidad y la observación no participante; los instrumentos utilizados fueron el temario de preguntas, diario de campo y fichas de observación. El equipo de investigación hizo el acercamiento con las madres y sus hijos e hijas, quienes de manera voluntaria decidieron participar en el estudio. Entre los principales hallazgos se evidencia que la interacción comunicacional entre madres e hijos está marcada por una relación cercana y con un tiempo adecuado para que las madres estén pendientes de sus hijos; sin embargo, la propia dinámica y entorno carcelario generan situaciones complejas de manejar para ambos. Se concluye que es fundamental la generación de espacios de acompañamiento para madres e hijos, de tal manera que la
\end{abstract}

\footnotetext{
${ }^{1}$ Universidad Técnica de Cotopaxi, Facultad de Ciencias Humanas y Educación, Latacunga, Ecuador, margarita.cortes0109@utc.edu.ec

${ }^{2}$ Universidad Técnica de Cotopaxi, Facultad de Ciencias Humanas y Educación, Latacunga, Ecuador, nathalie.ortiz3269@utc.edu.ec

${ }^{3}$ Universidad Técnica de Cotopaxi, Facultad de Ciencias Humanas y Educación, Latacunga, Ecuador, lourdes.cabrera@utc.edu.ec
} 
interacción entre los diversos actores del CRS, y principalmente, entre madres e hijos pueda ser un elemento favorecedor para relaciones armónicas basadas en el diálogo y la empatía.

Palabras claves: Comunicación, cárcel, Ecuador, habilidades, interacción, infancia, mujeres.

\begin{abstract}
.
The problem related to childhood and prison is a subject hardly analyzed from the communication field, so the objective of this article is to expose the processes of communicational interaction that occur between mothers and children that remain inside the Female Rehabilitation Social Center (RSC) of Quito, Ecuador. The methodological strategy was based on a non-experimental design, of a transversal nature, through a qualitative approach through the technique of in-depth interviews and non-participant observation; the instruments used were the questionnaire, field diary and observation sheets. The research team approached mothers and their sons and daughters, who voluntarily decided to participate in the study. The main findings it is evident that the communicational interaction between mothers and their children is marked by a close relationship and with an adequate time for mothers to be aware of their children; however, the dynamics and prison environment generate complex situations of handling for both. It is concluded that the creation of accompaniment spaces for mothers and their children is fundamental, so that the interaction between the actors of the RSC, and mainly, between mothers and children, can be a favorable element for harmonic relationships based on dialogue and empathy.
\end{abstract}

Keywords: Communication, jail, Ecuador, skills, interaction, childhood, women.

\title{
Introducción.
}

La libertad es el derecho que todo ser humano obtiene al nacer y es reconocido por la ley, pero cuando se irrespetan las leyes que dicta el Estado, el mayor castigo es la privación de la libertad, según Alarcón Granobles (como se citó en Vite y Reyes, 2016), la "privación de la libertad históricamente ha sido vista como una forma por excelencia para combatir los problemas sociales, culturales o sociológicos derivados de la delincuencia, además que genera un temor o miedo a tal punto que amedrenta a la comunidad" (p.1). En el caso de Latinoamérica, Argentina, Brasil, Bolivia, Chile, Colombia, Ecuador, Paraguay, Perú, Uruguay y Venezuela se encuentra el $9 \%$ de las personas privadas de la libertad (ppl), con unos 1.018 .846 ppl para el año 2014, según el último informe global de International Centre for Prision Studies.

Ecuador es uno de los países con menor tasa de encarcelamiento, a diferencia de otros países de América Latina, sin embargo, esto no implica la inexistencia de una realidad alarmante:

Los centros se han convertido en verdaderas bodegas humanas, donde se profundiza la violación de los derechos humanos de las madres e hijos toda esta transgresión ha llevado a la degradación del ser humano y va perdiendo la capacidad para vivir de manera digna. (Niebla, 2014, p.2) 
En Ecuador, los Centros de Rehabilitación Social están divididos en: Centros de Privación Provisional de Libertad, Centros de Rehabilitación Social de Media Seguridad, Mínima Seguridad y Máxima seguridad. De acuerdo a datos estadísticos obtenidos el 31 de marzo del 2013 en Ecuador existen:

107.269 personas condenadas en los distintos sistemas de privación de libertad, de ellos un $89 \%$ son hombre y un $11 \%$ mujeres y si consideramos los estudios de la misma institución del año 2008 cuando existían 81.086 en las mismas condiciones con un total de 31.777 niños y niñas que son hijos de personas que cumplen condena. (Niebla, 2014, p.4)

Una particularidad con respecto a las prisiones es que han sido creadas pensando, principalmente, en las necesidades y características de la población masculina, olvidándose de los requerimientos propios de las mujeres, sobre ello Medeiros (2015) afirma que:

El tratamiento brindado a las mujeres en el sistema penal es un reflejo de la posición social que les designa. Desafortunadamente, el sistema tiene una perspectiva androcéntrica y quienes no poseen el perfil que sostiene un modelo masculino hegemónico tendrán que adaptar sus necesidades a los modelos prevalecientes. Al largo de la historia las mujeres fueron tratadas socialmente dentro de un sistema de desigualdades y de devaluación siempre conectada a su condición sexual y biológica. El género femenino ha sido siempre designado en el contexto de la belleza, de la pureza, de la maternidad, en tanto procreadoras de los hijos y guardianas del hogar. (p.6)

La Convención sobre los Derechos del Niño, aprobada el 20 de noviembre de 1989 por la Asamblea General de Naciones Unidas, establece en su artículo 9 que ningún niño y niña deberá ser separado de sus padres, a menos que sea por su propio bien. En el caso de que su padre y madre estén separados tienen derecho a mantener contacto con sus hijos e hijas; pero, ¿qué sucede cuando el niño y la niña dependen de su madre mientras ella está privada de la libertad? ¿qué ocurre con sus hijos e hijas al tener que convivir con ellas en un centro de rehabilitación social? Aunque las personas privadas de la libertad son consideradas por parte del Estado ecuatoriano como un grupo de atención prioritaria, así lo estipula la Constitución desde el año 2008; sin embargo, a decir de Lora (2012) "no se garantiza en la máxima medida su posible desarrollo, debido a que se lo mantiene en un medio donde es vulnerable a sufrir perjuicios o abusos" (p.9).

Además de estos instrumentos legales internacionales, en el caso de Ecuador, existe una norma técnica de protección especial, servicios y programas familiares expedida por el Ministerio de Inclusión Económica y Social (MIES), la cual tiene como objetivo garantizar el bienestar y velar porque los derechos de ese grupo humano no sean vulnerados. Pese a estas iniciativas, uno de los retos que tiene el Estado ecuatoriano es "la implementación de un sistema penitenciario que garantice los derechos de las personas privadas de la libertad y que permita una rehabilitación e inserción de aquellas (os) que cometieron un error en sus vidas" (Villareal como se citó en Vite y Reyes, 2016).

En este contexto, la presente investigación se desarrolló de manera específica en el Centro de Rehabilitación Social (CRS) de Atención Prioritaria Femenino Quito, ubicado en el sector de 
Chillogallo, este centro recibe a mujeres con sentencia por delitos como tráfico de drogas, asociación ilícita u homicidios, el CRS está compuesto por 46 mujeres, 27 niñas,18 niños, 10 agentes penitenciarios, 6 educadoras, entre escuela y bachillerato, 10 trabajadores administrativos: 1 psicólogo, médico familiar, ginecólogo, dentista, nutricionista, entre otros, además este centro cuenta con una guardería que recibe a niños y niñas de entre 12 meses hasta los 3 años de edad, y su finalidad es contribuir al desarrollo de los infantes, cuenta con una coordinadora general y cinco educadoras, el lugar dispone de cuatro aulas, comedor, zonas para la estimulación temprana y espacios verdes para la recreación.

Muchas de las mujeres que cumplen su condena en este centro conviven con sus hijos e hijas los primeros tres años de vida, luego de esa edad los niños y niñas deben ser entregados a familiares de la persona privada de la libertad (ppl), o son enviados a casas asistenciales, excepto los niños y niñas que sufren de discapacidad, enfermedades catastróficas, maltrato y/o abandono por parte del adulto que en el ámbito externo está a cargo del niño o niña, en estos casos conviven con su madre hasta los 6 años o hasta que el CRS autorice la estadía del menor.

El objetivo general de este artículo es exponer los procesos de interacción comunicacional que se dan entre madres e infantes que conviven en el CRS de Atención Prioritaria Femenino de Chillogallo, como objetivos específicos se planteó: a) determinar las habilidades comunicacionales que desarrollan las madres al interior del CRS femenino de Quito; b) identificar las habilidades comunicacionales que desarrollan los niños y niñas que conviven al interior de centro de rehabilitación femenino de Quito; y, finalmente, el equipo de investigación se propuso c) diseñar estrategias orientadas al apoyo de madres e hijos que conviven al interior del CRS. Y la pregunta de investigación que orientó este trabajo fue: ¿Qué características tienen los procesos de interacción comunicacional entre madres e hijos que conviven en el Centro de Rehabilitación Social de Atención Prioritaria Femenino de Quito, ubicado en Chillogallo?

El equipo investigador consideró importante realizar esta investigación debido a que los derechos de las ppl son irrespetados tanto en el caso de mujeres, como de los hombres; sin embargo, la situación de las mujeres es particular debido a que gran parte de quienes permanecen en una cárcel son madres. En limitadas ocasiones esta problemática es analizada desde el ámbito comunicacional, por ello el interés fue indagar en los procesos de interacción comunicacional entre madre e hijos en un contexto de encierro carcelario. En una primera parte se hará referencia a los elementos fundamentales detectados en la revisión documental, posteriormente se detallará la estrategia metodológica, seguido de los resultados y discusión, finalmente se expondrán las conclusiones a las que arribó este estudio.

\section{Aproximaciones teóricas al concepto de interacción comunicativa}

El concepto fundamental de este proyecto son los procesos de interacción comunicacional los cuales pueden ser: lineales, circulares y reticulares; los lineales son aquellos que miran a la comunicación como un proceso en donde interviene un emisor y receptor; la comunicación vista desde una perspectiva circular implica la retroalimentación entre los seres humanos; y, la 
comunicación reticular es aquella que se da en red y en donde tiene lugar intercambio de conocimientos. (Carrillo, Hamit, Benjumea y Segura, 2017)

Las características que adopten los procesos de interacción de los seres humanos van a estar determinadas por el contexto familiar, sobre este aspecto Puello et al., (2014) señalan que la comunicación: "se basa en convenciones sociales y la realidad es un producto de la comunicación. Esta es muy importante para la familia por ser un proceso de interacción donde se construyen relaciones horizontales y verticales, se intercambian mensajes, informaciones, afectos, comportamientos" (p.7-8).

El núcleo familiar es el elemento básico para construir modos de comunicación y la calidad de la comunicación, entre padres/madres e hijos, incidirá en una interacción mayormente positiva o por el contrario marcada por constantes conflictos. Schmidt, Maglio, Messoulam, Molina y González (2010) señalan que "la comunicación familiar funcional permite el desarrollo de un auto concepto positivo" (p.1). De igual manera, en el núcleo familiar es importante la presencia de padre y madre porque son el factor protector y quienes generan reglas y optimizan un proceso adecuado de comunicación, pero cuando uno de ellos falta en el hogar los procesos de comunicación presentan muchos retos los cuales deben ser enfrentados para evitar procesos disfuncionales, en el caso de las familias que tienen a uno de sus miembros privados de la libertad, el escenario es complejo, Schmidt et al., (2010) explican que:

Las perturbaciones que dicha situación provoca específicamente en niños, niñas y adolescentes pertenecientes a familias con adultos privados de libertad, quienes deben enfrentarse a una vida compleja y cruel, soportando la presión del dedo acusador de la sociedad que no se detiene a hacer distinción entre las partes, sino más bien, clasifica a todo el núcleo familiar como uno solo, imponiendo duramente con el estigma social, una extensión de la condena a los consanguíneos. (p.3)

El proceso comunicacional es inherente al desarrollo de habilidades sociales, sus elementos intervinientes son las emociones, la cultura y el contexto social en el que aparecen; aspectos fundamentales para desarrollar y mantener una comunicación efectiva, la cual es vital para el desarrollo del ser humano (Hoyuelos, 2018). Comunicación es fundamentalmente interacción, Rizo (2006) señala que la interacción es "el principio básico de la organización social, y como tal, es requisito indispensable para las relaciones sociales" (Rizo,2006, p.2). La comunicación nos permite construirnos como seres sociales y a su vez posibilita la generación de lazos vitales que son los que otorgan sentido a la existencia, "la interacción es siempre comunicación con otro distinto a uno mismo" (Rizo, 2006, p.16), esto se verá condicionado por el contexto sociocultural en donde se produzca la interacción.

Los procesos de interacción son frecuentemente analizados desde el campo educativo, debido a que "la enseñanza formal es uno de los ámbitos de la vida social donde la expresión verbal, no verbal y paraverbal, tienen una presencia importante" (Herrero,2012, p.2). Los escenarios escolares son un espacio clave de encuentros y desencuentros y, por tanto, la comunicación es una dimensión fundamental para garantizar procesos educativos exitosos, sobre ello Herrero (2012) 
afirma que "es importante tener en cuenta tanto en el alumno como en el profesorado, sus características personales, contextuales, sus sentimientos, etc. que influirán de manera directa en la comunicación e interacción entre ambos" (p. 5).

Tal como se ve en estos planteamientos, el escenario en donde se produce la interacción es una característica interviniente y decisiva en los procesos de comunicación, precisamente González (2013) al explorar la comunicación interpersonal determina varias dimensiones: inicio, conservación y desarticulación; y bajo estas etapas examina y discute las formas de comunicación humana que se producen a diario, experiencias que se producen en la familia, círculo de amigos, pareja, instituciones educativas, compañeros de clase, espacio laboral, entre otros, "quienes somos depende de la comunicación, porque descubrimos nuestra identidad" (González, 2013, p.9).

\section{Metodología.}

\section{Tipo de diseño.}

La investigación se basó en un diseño no experimental de tipo transversal, el cual, según Sousa, Driessnack y Costa (2007) "no tienen determinación aleatoria, manipulación de variables o grupos de comparación. El investigador observa lo que ocurre de forma natural, sin intervenir de manera alguna" (s. p).

\section{Enfoque.}

En esta investigación se usó el enfoque cualitativo, según Cadena, et al (2017) el investigador cualitativo "ve al escenario y personas en una perspectiva holística, las personas, escenarios o grupos no son reducidos a variables, sino vistos como un todo" (p.4).

\section{Población y muestra.}

El CRS de atención prioritaria de Quito está conformado por 46 mujeres ppl y 45 niños y niñas. La muestra estuvo conformada por 10 participantes: 5 madres, quienes tenían entre 27 y 42 años; y, sus hijos e hijas cuyas edades fluctúan entre 4 meses y 11 años de edad (Tabla 1 y 2). Se utilizó un muestreo no probabilístico, el cual "permite seleccionar muestras con una clara intensión o por un criterio preestablecido. Las muestras que se seleccionan buscan, desde luego, una representatividad de la población" (Niño, 2011, p.57). En esta investigación se aplicó el muestreo de voluntarios, es decir, las personas de manera libre decidieron ser parte de la investigación.

Tabla 1. Criterios de inclusión de la muestra

\begin{tabular}{cc}
\hline Criterios de Inclusión & Criterios de exclusión \\
\hline Madres que conviven con sus hijos & Mujeres sin hijos \\
Madres que estén en el CRS de Chillogallo al & Madres que estén 1 mes en el CRS de \\
menos 3 meses. & Chillogallo \\
Niños y Niñas que vivan en el CRS de & Niños y niñas que vivan en el CRS de \\
Chillogallo al menos 3 meses & Chillogallo de 1 a 2 meses
\end{tabular}


Madres en estado de gestación

Madres que estén separadas de sus hijos

Fuente: Elaboración propia

Tabla 2. Datos informativos de los participantes en el estudio

\begin{tabular}{ccccccccc}
\hline Código & $\begin{array}{c}\text { Eda } \\
\mathbf{d}\end{array}$ & $\begin{array}{c}\text { Tiempo } \\
\text { en el } \\
\text { CRS }\end{array}$ & $\begin{array}{c}\mathbf{N}^{\circ} \\
\text { de } \\
\text { hijos }\end{array}$ & $\begin{array}{c}\text { Edad de los } \\
\text { niños y niñas }\end{array}$ & Pareja & $\begin{array}{c}\text { Apoyo } \\
\text { de la } \\
\text { Familia }\end{array}$ & $\begin{array}{c}\text { Razón } \\
\text { del } \\
\text { encierro }\end{array}$ & $\begin{array}{c}\text { Código } \\
\text { niños/as }\end{array}$ \\
\hline 2RA6 & 26 & 7 meses & 1 & 4 meses & No & $\mathrm{Si}$ & $\begin{array}{c}\text { Homicidi } \\
\text { o }\end{array}$ & P4E \\
3GJ5 & 35 & 3 meses & 4 & $\begin{array}{c}14,10,8 \text { y } 1 \\
\text { año } 5 \text { meses }\end{array}$ & No & Si & $\begin{array}{c}\text { Tráfico de } \\
\text { drogas }\end{array}$ & J15 \\
3DJ6 & 36 & 2 años & 7 & $\begin{array}{c}22,18,15,9,7,6, \\
2 \text { años }\end{array}$ & No & No & $\begin{array}{c}\text { Tráfico de } \\
\text { drogas }\end{array}$ & D6M \\
4LM2 & 42 & 3 años & 2 & $\begin{array}{c}24 \text { y 2 años } 4 \\
\text { meses }\end{array}$ & No & No & $\begin{array}{c}\text { Homplicidi } \\
\text { de }\end{array}$ & L24 \\
2MA7 & 27 & 4 años & 3 & 11,9, y 4 años & No & Si & $\begin{array}{c}\text { Tráfico de } \\
\text { drogas }\end{array}$ & M4A \\
\hline
\end{tabular}

Fuente: Elaboración propia

\section{Técnicas e instrumentos}

La estrategia metodológica para recolectar la información se apoyó en el método de la observación no participante, la cual se define como aquella que "recoge la información desde afuera, sin intervenir para nada en el grupo social, hecho o fenómeno investigado" (Huamán, 2005, p.16), la observación se la realizó durante 7 días al interior del CRS, el objetivo fue recabar información de primera mano y así poder identificar las habilidades comunicacionales que poseen los infantes. Además, se usó la técnica de la entrevista en profundidad con las madres participantes, la finalidad fue recabar elementos que permitan establecer los momentos más trascendentales para la historia de vida de las mujeres.

La aplicación de estas técnicas permitió determinar cómo se dan los procesos de interacción comunicacional a través del rastreo de las habilidades comunicacionales tanto en las madres como en sus hijos.

Los instrumentos, en el caso de la observación no participante, fueron el diario de campo y una ficha de observación (Tabla 3 y 4) para cada uno de los hijos de las mujeres; y para las entrevistas en profundidad se diseñó un temario de preguntas vinculado a las dimensiones de cada objetivo específico.

Tabla 3. Elementos del diario de campo

$$
\text { Fecha: }
$$


ISSN: 2600-5859

Vol. 3, N4, p. 102-121, octubre-diciembre, 2020

Actividad:

Participantes:

Descripción de la actividad

Interpretación (reflexión sobre lo observado)

Fuente: Elaboración propia

Tabla 4: Ficha de observación

\begin{tabular}{|l|l|}
\hline Espacio observado: & \\
\hline Nombre del niño/a: & \\
\hline Nombre de la madre & \\
\hline Fecha: & \\
\hline Hora: & \\
\hline
\end{tabular}

\begin{tabular}{|c|c|c|c|c|c|}
\hline & Indicadores & Nunca & $\begin{array}{l}\text { Algunas } \\
\text { veces }\end{array}$ & Frecuentemente & Observaciones \\
\hline 1 & $\begin{array}{l}\text { Saluda y/o sonríe a las personas } \\
\text { cercanas a su entorno }\end{array}$ & & & & \\
\hline 2 & $\begin{array}{l}\text { Es capaz de decir su nombre } \\
\text { cuando se lo preguntan }\end{array}$ & & & & \\
\hline 3 & $\begin{array}{l}\text { Dice gracias cuando se relaciona } \\
\text { con otros niños o adultos }\end{array}$ & & & & \\
\hline 4 & $\begin{array}{l}\text { Se acerca a otros niños o niñas } \\
\text { para participar de un juego o para } \\
\text { conversar }\end{array}$ & & & & \\
\hline 5 & $\begin{array}{l}\text { Se adapta fácilmente a los juegos } \\
\text { o actividades que otros niños o } \\
\text { niñas ya han iniciado }\end{array}$ & & & & \\
\hline 6 & $\begin{array}{l}\text { Si un niño o niña hace algo que le } \\
\text { desagrada le expresa su } \\
\text { descontento }\end{array}$ & & & & \\
\hline 7 & $\begin{array}{l}\text { Dice gracias cuando se relaciona } \\
\text { con su madre }\end{array}$ & & & & \\
\hline 8 & $\begin{array}{l}\text { Demuestra afecto y cariño a su } \\
\text { madre }\end{array}$ & & & & \\
\hline 9 & $\begin{array}{l}\text { Es capaz de responder a una } \\
\text { inquietud sencilla de un adulto }\end{array}$ & & & & \\
\hline 10 & $\begin{array}{l}\text { Quiere saber el porqué } \\
\text { algunas situaciones y les } \\
\text { pregunta a los adultos }\end{array}$ & & & & \\
\hline
\end{tabular}

Fuente: Recuperado de "Habilidades sociales preescolares: una escala para niños de contextos de pobreza Lacunza, Castro y Contini, p.26., 2009.

\section{Resultados y discusión.}


Una vez que se aplicó la metodología se procedió a transcribir la información obtenida de las entrevistas en profundidad y se sistematizó la información del diario de campo; posterior a ello se procedió a identificar las principales categorías vinculadas a los elementos centrales de los objetivos: a) habilidades comunicacionales de las madres, b) habilidades comunicacionales de los niños y niñas; y, c) Estrategias orientadas al apoyo de madres e hijos; en ese sentido el análisis de los resultados se presentará en función de esas dimensiones.

\section{Habilidades comunicacionales de las madres.}

Un elemento importante que apareció con la aplicación de las técnicas de investigación es respecto al vínculo madre-hijo, el cual revela aspectos fundamentales sobre las habilidades comunicacionales que se desarrollan al interior del CRS, las madres aseguran que la relación entre ellos ha mejorado, es decir, que aseguran tener un lazo más fuerte, porque pasan juntos las 24 horas del día, lo que no ocurría cuando ellas estaban fuera del CRS, los quehaceres de la casa y el trabajo les impedían estar pendientes de sus hijos, una de las madres relató lo siguiente: "nunca me había sentido tan unida como lo estoy ahora con mi hijo, ahora siento más responsabilidad con mi hijo ya que afuera uno ya está haciendo muchas cosas y nos descuidamos de nuestros hijos" (3GJ5, comunicación personal, diciembre 2019). Como se expresa en esta situación un factor fundamental para el desarrollo de las habilidades comunicativas tiene que ver con los momentos para la interacción, al respecto O’Connor y Seymour (1999) (como se citó en Batista y Romero, 2007) señalan que:

Estas habilidades están representadas por las capacidades de desempeñar determinadas tareas comunicacionales de modo consistente para influir en las personas, pues la comunicación es un círculo donde el sujeto influye en otros individuos y los otros en él. Por lo tanto, es necesario dominarlas para afrontar los diversos cambios que se presentan. (p. 39)

A pesar de la situación y el entorno en donde se desarrolla este grupo vulnerable, las madres buscan estrategias para comunicarse con sus hijos y de esta manera crear una comunicación positiva para que los niños y niñas no sientan la falta de libertad, tomando en cuenta que en los primeros años de vida se crea la identidad del infante.

Por otro lado, es importante tener en cuenta que la mayoría de las mujeres en este centro han tenido que afrontar varios retos y desafíos, uno de ellos es aprender a controlar su carácter para evitar confrontarse con sus compañeras:

Es difícil aprender a convivir con mis compañeras, no hay respeto cuando los niños están durmiendo en el pabellón ellas gritan, dicen malas palabras juegan y todo, pero si se les reclama ellas salen más bravas y nos dicen que esto no es un hotel (2RA6, comunicación personal, diciembre 2019)

La experiencia de esta madre es muy recurrente en todas las mujeres entrevistadas, porque estas situaciones suceden las 24 horas del día en los 4 pabellones de CRS, también se pudo evidenciar, a través del diario de campo, que es usual que se generen comentarios negativos de otras mujeres, lo cual se constituye en una causa para los conflictos al interior del CRS, elemento 
contraproducente para la generación de una interacción comunicacional adecuada, al respecto Batista y Romero (2007) señalan que es importante que el ser humano desarrolle "habilidades comunicativas, a saber: escuchar activamente, hablar con claridad, habilidad para responder, agudeza sensorial (visual, auditiva, tacto, gusto y olfato) flexibilidad, asertividad, empatía y sinergia"(p.38). Sin embargo, el contexto del encierro carcelario es una variable que limita en gran medida tal situación.

En todo tipo de escenarios, y más aún en una prisión, es importante trabajar en el fomento de acciones que posibiliten el desarrollo de habilidades comunicacionales, Tejera y Cardoso (2015) explican que:

Habilidades para comunicarse y desarrollarlas, constituye un importante factor de adaptación social, toda vez que contribuye al perfeccionamiento de la vida de relación del hombre, a dar calidad a las diferentes actividades que realiza y mejorar sus modos de actuación en el contexto donde le ha tocado vivir. (p.171)

En efecto, las dinámicas propias de un centro carcelario le plantean varios retos a las madres a la hora de intentar desarrollar las habilidades comunicacionales que requiere ese entorno. Otro aspecto que se evidencia tiene que ver con la manera en que fechas como los cumpleaños se convierten en un momento fundamental para los procesos de interacción entre madres e hijos. La mayoría de las mujeres ha festejado el cumpleaños de su hijas o hijos dentro de la cárcel, el encierro no ha sido un impedimento para festejar un día importante en sus vidas: "aquí yo le he festejado sus 4 añitos mandé a comprar pastel y colas, pero este último año le hice una mesita completa con bocaditos, colas, piñata y pastel, mi hija estuvo muy feliz" (2MA7, comunicación personal, diciembre 2019).

El CRS permite estas actividades porque las considera beneficiosas para el desarrollo de la personalidad del niño o niña, además, algunas fundaciones se hacen presentes en fechas especiales como la navidad o el día del niño, brindan un espectáculo exclusivamente para los hijos de las mujeres privadas de la libertad y de este modo poder entretener a los niños, al menos, por unas horas: "varias fundaciones llegaron y realizaron programas donde los niños podían participar eran programas solo para ellos, donde entregaban regalos y podíamos ver la felicidad de nuestros hijos al ver sus regalos y poder compartir con otras personas" (3GJ5, comunicación personal, diciembre 2019).

Estos hallazgos son coincidentes con lo propuesto por Tejera y Cardoso (2015) quienes explican que unas de las habilidades comunicativas son aquellas que promueven la relación empática, la cual está relacionada con el fomento de diversas acciones orientadas a la construcción de experiencias. Los componentes de esta habilidad son el "acercamiento afectivo. Expresar una actitud de aceptación, de apoyo, dar posibilidad de expresión de vivencias del otro" (Tejera y Cardoso, 2015, p. 170). 
Este tipo de experiencias son vitales para que las mujeres puedan manejar y sostener sus habilidades comunicativas dentro del CRS, aunque hay que tomar en cuenta que en los casos analizados todas provienen de entornos marcados por la violencia y pobreza:

A los 19 años yo di a luz a mi primer hijo el papá de mi niño desapareció y la necesidad me obligó a vender droga por mi barrio... yo vendía poco, lo necesario para sobrevivir luego llegaron mis otros 2 hijos y yo seguía en el mismo trabajo, esto no era nada fácil porque no podía estar tranquila en ningún lado...porque temía a que en cualquier lugar ya me iban a coger presa. (3GJ5, Comunicación personal, diciembre 2019)

Entonces, se puede afirmar que para las mujeres que conviven al interior del CRS, debido a sus antecedentes de exclusión y estigmatización, trabajar en sus habilidades comunicativas es todo un desafío, esto es convergente con lo afirmado por Dos Santos y Benavides (2014) (como se citó en Flores, García, Calsina y Yapuchura, 2016) "Pero, en situaciones hostiles, cuanto más agresión recibe la persona, menores son sus habilidades sociales relacionadas con la asertividad y con su repertorio general; así también tienen una menor capacidad empática en relación al factor altruismo" (p. 4).

El CRS cuenta con 4 pabellones (A, B, C y D), el pabellón D presentó una peculiaridad: las madres que ahí conviven fomentaron la creación de un espacio dedicado, exclusivamente, al entretenimiento de sus hijos. Todos los días, en el horario de $18 \mathrm{~h} 00$ a 19h00 pm, los infantes pueden mirar programas televisivos infantiles, así como desarrollar actividades lúdica. Precisamente, promover la participación de los diversos sujetos involucrados en una situación genera mejores condiciones para un ambiente de respeto, así lo proponen Tejera y Cardoso (2015 ) "Establecer una adecuada comunicación sobre la base de la comprensión mutua y el respeto a la personalidad del sujeto" (p.169).

En general, en esta actividad los niños/as se muestran contentos y relajados, excepto una de las niñas, quien necesariamente debe estar con su madre para controlar su comportamiento agresivo, la niña tienen dificultades para una comunicación asertiva, lo cual se explica por el ambiente en el que se ha desarrollado, el cual no le ha permitido desplegar una actitud consciente, esto es convergente con los planteamientos de González-Moreno (2012) quienes explican que la actividad reflexiva "es la capacidad de reformular el propio pensamiento, mediante el uso del lenguaje, lo que lleva a reflexionar sobre el comportamiento de sí mismo y las acciones de los demás” (p. 597)

Frente a la necesidad de la madre de comunicarse con sus hijos, esta se torna un tanto compleja debido a que los niños/as no han desarrollado las habilidades comunicacionales propias de su edad; sin embargo, de acuerdo a la experiencia de las madres pese a ello encuentran formas para comprender cuando su niño/a necesita algo, porque todo signo o gesto comunica, es decir el principal mecanismo es el lenguaje no verbal, a continuación el extracto de un relato:

Mi hija aún no puede hablar y además ella tiene un carácter muy fuerte, así que si necesita algo solo me señala y yo le doy lo que ella quiera, a pesar que ella es muy independiente, ni cuando 
está peleando me necesita, mi hija tiene 2 años 4 meses y aún toma el seno y cuando ella quiere lactar simplemente, saca mi seno y ya". (4LM2, comunicación personal, diciembre 2019)

La convivencia durante las 24 horas del día les ha permitido desplegar habilidades para satisfacer las necesidades de sus hijos, aunque también existen varios factores que impiden una comunicación eficaz, el principal es el contexto del encierro, pero este no ha sido impedimento para buscar mecanismos de comunicación por parte de las madres.

\section{Habilidades comunicacionales de los niños y niñas.}

A través de la aplicación de método de la observación, orientado a la dimensión del segundo objetivo específico, el cual busca identificar las habilidades comunicacionales que han desarrollado los niños y niñas al interior del CRS, se pudo evidenciar varias dificultades para que los infantes puedan comunicarse verbalmente con las personas de su entorno; la principal forma de comunicación es mediante el lenguaje no verbal. Aunque al interior de la guardería las profesoras acompañan al proceso de enseñanza aprendizaje este no obtiene resultados favorables porque no es reforzado por la madre, quien carece del conocimiento adecuado para promover ese tipo de habilidades.

Sobre las limitantes que presenta el habitar en una cárcel, una de las madres relató lo siguiente:

Yo siento que mi hijo se estresa mucho, porque cuando está aquí no para de llorar, duerme muy poco, se asusta con facilidad... se reniega, en cambio cuando él sale con mi hermana por unos días ella me dice que pasa tranquilo, él solo pasa el portón negro y su estado de ánimo cambia totalmente a pesar de que es muy pequeño yo siento que el pasa mal aquí” (2RA6, Comunicación personal, diciembre 2019)

Este es el único caso en donde el niño es menor de un año, específicamente tiene 7 meses, los otros niños y niñas oscilan entre los 17 meses y 6 años de edad. Estos mecanismos de comunicación es lo que García (1995) denomina como la etapa prelocutoria, la cual se presenta en los primeros meses de vida en donde el niño/a no puede comunicarse mediante palabras y cuando tiene alguna necesidad la comunica a través del llanto.

A través del diario de campo se pudo observar a cinco niños/as de diferentes edades como: J15 tiene 1 año 5 meses, J2Y tiene 2 años, D6M tiene 6 años, M4A tiene 4 años y L24 tiene 2 años 4 meses, ellos pasan de 8:00 am a 15:30 pm en la guardería, logramos identificar que tienen diferentes formas para comunicarse, a pesar que su lenguaje oral no es muy fluido en comparación con otros menores de su misma edad, intentan transmitir un mensaje a través del habla y lo complementan con gestos y señales desarrollando habilidades como: aprender a escuchar, agradecer, hablar con amabilidad y firmeza. Pérez y Salmerón (2006) afirman que desde los 12 hasta los 24 meses los niños y niñas desarrollan "estrategias de aproximación a las palabras" (p.115), para los niños y niñas de entre 24 y 36 meses su lenguaje oral es más comprensible: "Cada vez se le va entendiendo mejor, aunque todavía puedan darse errores propios del habla infantil como por ejemplo dificultades con la /d/, la /o/ y la /s/ y con la /r/ y la /rr/, y la simplificación de sinfones y diptongos" (Pérez y Salmerón,2006, p.115). 
Por varios días logramos observar que M4A cambia la pronunciación de las palabras, por ejemplo, en vez de "si" dice "ti", esta situación se repite con L24, como mencionan los autores esto es normal según la edad de los niños y niñas; por otro lado, J2Y tiene dos años pero en este caso ella dialoga únicamente con una persona adulta de confianza, no habla mucho con sus compañeras de aula, y si necesita algo solo se acerca a su profesora, en caso de que existan personas nuevas en el lugar se intimida y evita participar con el grupo. Al ingresar al aula, una de sus profesoras dio una orden para que todas las niñas que estaban ahí saluden, pero ella fue la única que no dijo nada, ni tampoco se acercó, a diferencia de las otras niñas, quienes sí cumplieron con el pedido.

Por las características que tiene el CRS los niños y niñas deben aprender a convivir con los guías penitenciarios, quienes portan un uniforme distintivo, se observó una interacción con los infantes caracterizada por muestras de afecto, D6M tiene 6 años, la niña más grande del centro, cuando ve a un guía entrar a los patios ella corre, la abraza y los guías le corresponden a la acción de la niña. Hay que tomar en cuenta que los niños y niñas son prioridad en esta casa de acogida, es por esto que se tiene totalmente prohibido el maltrato, en cualquiera de sus formas, por parte de sus madres, cuando los guías observan ese tipo de actos realizan partes para sancionar a las mujeres con el fin de proteger la integridad de los infantes.

Por otro lado, los niños y niñas a su corta edad asumen que los guías penitenciaron son autoridad dentro del CRS, es peculiar observar cómo imitan las acciones de los guías, L24 tiene 2 años 4 meses y a su corta edad ya conoce las actividades diarias del centro, un día mientras ella jugaba los guías gritaban con fuerza "señoras... el rancho"(refiriéndose a la comida), ella automáticamente dejó de jugar, se paró en la mesa y gritó "el ancho" (el rancho), sobre ello Peralta (2000) sostiene que: "desde los 18 a los 24 meses, el niño aprende a involucrarse en un diálogo, adoptando y asignando roles comunicativos. El niño ha aprendido a dar significado y en adelante podrá extender el rango de las significaciones" (p.60).

En la mayoría de los casos investigados se pudo evidenciar que las madres no permiten que sus familiares vayan a visitarles, este es el caso de 4LM2, 2MA7, 2RA6, entre las razones que mencionaron fue: "es mejor que la familia no vea la realidad en la que vivimos así evitamos que nuestros hijos se encariñen con las visitas y sufran"; por otro lado 3DJ6 disfruta la visita de sus hijos, ella nos comentaba que uno de sus hijos le visita cada 15 días, para sus hijas menores es una figura paterna y le llaman papá, un rasgo particular en este caso es que a una de sus hijas (J2Y) no le gusta compartir con las visitas y prefiere estar en el pabellón. Por otro lado, D6M espera ansiosa los días para ver a su hermano, para ella es su "papá Juan", "me siento muy feliz cuando mi papi viene, paso jugando todo el día con él, le cuento todo lo que ha pasado y le digo que le quiero mucho, que ya mismo vamos a salir de aquí para estar en familia y comer todos juntos" (D6M, comunicación personal, diciembre 2019).

Otro elemento que se priorizó para el análisis fue las formas de interacción que se dan entre los niños y niñas que conviven al interior del CRS, en los 7 días de observación se logró identificar que en la guardería se comportan de una manera y cuando salen de ella, es decir, cuando permanecen con sus madres, se presentan otras formas. En la guardería, demuestran una relación 
solidaria, respetuosa y de amistad, precisamente una de las acciones de su profesora es fomentar la amabilidad a la hora de dialogar; sin embargo, en cuando están con sus madres, aparentemente, olvidan lo aprendido en el aula de clase y muestran actitudes de rebeldía.

Si bien, hay elementos comunes que comparten los niños y niñas en el CRS, también tienen sus peculiaridades, por ejemplo en el caso de J2Y se muestra tímida, seria y solitaria, M4A es cariñosa, participativa, risueña y sociable, L24 es seria, divertida, solidaria y respetuosa, mientras está en la guardería; pero, en el pabellón es agresiva con los niños más pequeños, hace rabietas y se acuesta en el piso, es por esto que el objetivo de la guardería es fomentar una actitud de diálogo y respeto "la conciencia de que cada persona que encuentran a su paso es también un ser humano como ellos mismos. A partir del logro de dicha perspectiva, el niño puede comprender que sus acciones producen placer o sufrimiento en los otros" (Mcginnis y Goldstein, 1990, p.6).

L24 muestra estos rasgos agresivos y llama la atención la forma en que busca conseguir sus objetivos, por ejemplo, ella quería sentarse en un lugar que estaba ocupado por otra niña, así que tomó su juguete y le dio a entender que quería jugar y lo arrojó lejos, entonces la niña que ocupaba el puesto salió corriendo por su juguete, mientras que L24 corrió a sentarse en el espacio que quería inicialmente. Estas acciones implementadas por la niña, pueden ser entendidas como habilidades que implican "un conjunto de conductas aprendidas y lo social, aporta lo impersonal" (Flores et al., 2016, p.6) Cada uno de los casos fue diferente, cada niño o niña crea sus propias habilidades para comunicarse, ya sea mediante lo aprendido en la guardería o de acuerdo al ambiente en el que se desenvuelve, transmitiendo lo que mira y vive en su día a día.

Varias de las mujeres evitan que los niños jueguen y prefieren tenerlos a su lado para impedir peleas y conflictos con sus madres, a D6M su madre le prohíbe salir a jugar con otras niñas porque asegura que pueden reñir y eso trae consigo que sus madres salgan en su defensa, lo cual genera discordias entre ellas: "yo por mis hijos me hago hasta matar, y si es de quedarme unos días más acá dentro pues me quedo porque nadie se mete con mis hijos" (2MA7, comunicación personal, diciembre 2019). Respecto de la sobreprotección que ejercen las madres, Pérez y Salmerón (2006) afirman que:

Sobreprotección, en la cual se sustituye continuamente al niño, no se le posibilita el contacto con elementos que generen experiencias de aprendizaje, el adulto se anticipa a sus necesidades, envolviendo al niño en un ambiente que no le deja interaccionar activamente; se propicia, en este contexto, un retraso en el lenguaje tanto a nivel pragmático como receptivo y expresivo. (p.119)

En general, los niños y niñas mantienen una relación respetuosa con las mujeres internas, cuando hay altercados entre los infantes ellas no les reclaman, al contrario, acuden directamente donde las madres. Entre los acuerdos que existen entre las madres es tratar de crear un ambiente de familia, por ejemplo, cuando van a clases y sus hijos se quedan en el pabellón a dormir piden ayuda a sus compañeras para que les vigilen mientras se desocupan: "la mayoría de las chicas se llevan bien con los niños, hasta el momento no se ha escuchado casos donde las mujeres maltratan a los hijos de otras compañeras" (2MA7, Comunicación personal, diciembre 2019). 
Otra de las acciones que se han generado en el CRS con la finalidad de crear un escenario propicio para los niños/as es tratar de erradicar las acciones violentas, en caso de que un niño/a maltrate a otro, se le explica y se le motiva para que no reaccione de la misma manera, se le solicita que acuda a la madre del niño/a y que le cuente lo sucedido para que sea ella quien reprenda la actitud violenta.

Mediante el diario de campo se pudo observar que D6M mantiene una buena relación con la mayoría de las mujeres, ella se sienta y le gusta participar de las conversaciones:

Mi hija [D6M] es como una persona adulta, conversa con todas mis compañeras es muy educada porque ella sabe que si se porta mal le doy dos nalgadas, aunque ella me sabe decir: verás mama si me pegas te van a sancionar y le digo así me sancionen yo te hago entender que es bueno y que no. (3DJ6, comunicación personal, diciembre 2019)

Otro elemento importante que se destaca en los niños/as es la incorporación del lenguaje carcelario, precisamente al referirse al hecho de que una de las madres pronto saldría en libertad, D6M dijo que ella iba a "salir en seco", al preguntarle sobre el significado de esa expresión aclaró que es "salir sin hacer papeles", esta forma de comunicación se evidencia en sus contactos cotidianos, los niños/as están muy familiarizados con las acciones y situaciones que se dan en el CRS; al preguntarle si conocía las razones por las que permanece ahí, sin temor alguno, dijo: "yo estoy aquí porque mi papá me dejó en la calle así que mi mamá hizo todo lo posible por traerme a vivir con ella, yo sé porque mi mamá está aquí por vender droga y por eso le dieron 3 años de cárcel” (D6M, comunicación personal, diciembre 2019) en concordancia con esta experiencia Gonzáles (como se citó en Flores et al., 2016) señala a "las habilidades sociales como un conjunto de comportamientos aprendidos que nos facilitan la consecución de refuerzo en situaciones de interacción. Esta, describe un conjunto de conductas en términos de su utilidad" (p.7).

\section{Estrategias de apoyo.}

El tercer eje alrededor del cual se planteó esta investigación fueron las estrategias de apoyo que se pudiesen implementar para apoyar a este grupo humano, precisamente para contar con mayores elementos se les preguntó a las madres acerca de las acciones que consideran necesarias para implementar en el CRS. A través de las entrevistas en profundidad, las mujeres privadas de la libertad, coincidieron en señalar que se debería implementar talleres de recreación los fines de semana, tanto para ellas como para sus hijos/as, de esta manera se mantendrían ocupadas y se podría enfrentar el estrés y la ansiedad que genera el encierro; además se pudo identificar que pese a los acuerdos y acciones implementadas tanto por las madres como por el CRS, existen dificultades que les impiden mantener una comunicación eficaz y generar un ambiente armónico, por ello es necesario trabajar en actividades para fomentar la asertividad porque cuando las personas desarrollan "habilidades sociales presentan un mayor número de miembros en su red social y tienden a mantener contactos más frecuentes con ellos” Jiménez, Vázquez y Vázquez (como se citó en Flores et al., 2016, p.7). Un mejor ambiente posibilitaría que niños y niñas enfrenten de mejor manera el encierro y los ayudaría para el desarrollo de habilidades porque estas 
"no son un rasgo de la personalidad, sino un conjunto de comportamientos aprendidos y adquiridos" (Flores et al., 2016, p.7).

Para las mujeres sus hijos/as son lo más importante y en todos los casos investigados esperan que el futuro sea diferente para ellos, aspiran a que sus hijos culminen sus estudios y sean profesionales, una de ellas relataba: "quiero verles a mis hijos triunfar que sean buenos profesionales y no unos mediocres que jamás se dejen ganar por las malas influencias y vayan por el camino del bien, que no sigan los pasos de su madre" (2MA7, comunicación personal, diciembre 2019) este testimonio se repite en todas las madres y señalaron que al salir del CRS serán otras personas para poder acompañar al desarrollo de sus hijos/as. Para que estas aspiraciones puedan concretarse es crucial el apoyo del Estado, a través de una política pública orientada al desarrollo de habilidades durante el encierro y estrategias de soporte posterior al encierro.

Así mismo, se pudo evidenciar que cada uno de los niños/as crean habilidades para comunicarse, pero una de las limitaciones que se presentan es la sobreprotección por parte de sus madres, lo cual impide un adecuado desarrollo de sus habilidades comunicacionales, sobre esto Pérez y Salmerón (2006) aseguran que: "sustituye continuamente al niño, no se le posibilita el contacto con elementos que generen experiencias de aprendizaje, el adulto se anticipa a sus necesidades, envolviendo al niño en un ambiente que no le deja interaccionar activamente" (p.119). Por las características del centro las madres se aferran al cuidado de sus hijos, y cuando hay discordias entre niños ellas actúan con violencia, esto causa que los menores transmitan lo que observan y se imposibilitan el desarrollo de habilidades como la empatía.

El equipo investigador, a partir de los datos obtenidos en esta investigación, realizará talleres de acompañamiento para el manejo de conflictos y estrategias de comunicación asertiva, concordamos con lo planteado por González (2013) quien señala:

La mayoría de las personas piensa que nacemos comunicándonos. Sin embargo, si comunicación significa "poner en común", entender lo que la otra persona me está diciendo, aprender a comunicarse es como aprender a caminar, a andar en bicicleta, manejar un auto. Aprendemos que cuando nos comunicamos, tenemos un impacto y este puede ser positivo o negativo. (s.p)

\section{Conclusiones.}

- Respecto a lo expuesto sobre los procesos de interacción entre madres e hijos al interior de un centro carcelario, se ha visto que estas dinámicas se ven determinadas, en el caso de las madres, por los escenarios previos caracterizados por la violencia en sus distintas manifestaciones. Tal como se ha visto las mujeres provienen de entornos altamente hostiles y, por tanto, sus habilidades comunicacionales están fragmentadas, lo cual entorpece procesos de interacción adecuados con sus compañeras; la relación con sus hijos se caracteriza por la sobreprotección, pese a ello el trabajo de campo también detectó acciones interesantes manejadas por las madres de uno de los pabellones y en donde el factor que las unió fue el bienestar de sus hijos/as. Una temática que deja abierta la investigación y que no fue una dimensión de análisis es cómo el ser parte de 
los procesos educativos, que ofrece el CRS a las mujeres, podría ser generador de ciertas habilidades al momento de comunicarse.

- Con relación a los procesos de interacción de niños y niñas se pudo notar que el lenguaje que se maneja en el CRS es interiorizado por ellos y es parte de su vida cotidiana; además en muchos casos, no han desarrollado las habilidades que requiere su edad, de tal manera que es complicado comunicarse con los sujetos de su entorno. Un hallazgo importante es ver cómo sus procesos de interacción cambian cuando están en la guardería y cuando están al cuidado de sus madres; además la interacción con los agentes penitenciarios es respetuosa e incluso se pudo evidenciar muestras de afecto.

- Y finalmente, las estrategias de apoyo son un requisito fundamental para que la convivencia mejore, es necesario elaborar acciones de acompañamiento cuyo eje sea la madre y su hijo/a, se podrían potencializar muchas características de las mujeres, y de igual manera se podría aprovechar la motivación que genera en ellas sus hijos e hijas.

\section{Referencias Bibliográficas.}

Batista, J., y Romero, M. (2007, septiembre-diciembre). Habilidades comunicativas del líder en universidades privadas y su relación con la programación neurolingüística. Revista de educación Laurus. 13(25), 36-64.

Cadena, P., Rendón, R., Aguilar, J.,Salinas, E.,De la Cruz, F. y Sangerman,D. (2017). Métodos cuantitativos, métodos cualitativos o su combinación en la investigación: un acercamiento en las ciencias sociales. Revista Mexicana de Ciencias Agrícolas, 8(7), 1603-1617.

Carrillo, M., Hamit, A., Benjumea, D., y Segura, M. (2017). Conceptualización de la interacción comunicativa y su caracterización. Revista Med, 25(2), 105-116.

Flores, E., García, M., Calsina, W., y Yapuchura, A. (2016). Las habilidades sociales y la comunicación interpersonal de los estudiantes de la Universidad Nacional del Altiplano. Revista de investigación en comunicación y desarrollo, 7(2), 5-14.

García,M. (1995). Comunicación y relaciones interpersonales. Portal de revistas electrónicas Universidad Autónoma de Madrid, 1133-2654.doi:1015366/tp.

González-Moreno, Claudia Ximena (2012). Formación del pensamiento reflexivo en estudiantes universitarios. Magis. Revista Internacional de Investigación en Educación, 4(9),595617.[fecha de Consulta 26 de Enero de 2020]. ISSN: 2027-1174. Disponible en: https://www.redalyc.org/articulo.oa?id=2810/281022848005

González, M. (Ed.). (2013).Comunicación interpersonal: inicio, mantenimiento y desintegración de relaciones. Monterrey, México: Editorial digital tecnológico de Monterrey. 
Hoyuelos, A. (2018). La comunicación en las relaciones sociales. Recuperado de https://www.macmillaneducation.es/wpcontent/uploads/2018/10/habilidades_sociales_libroalumno_unidad1muestra.pdf

Huamán, H. (2005). Manual de técnicas de investigación: conceptos y aplicaciones. Recuperado de https://books.google.com.ec/books?id=OEHABAAAQBAJ\&printsec=frontcover\&hl=es $\&$ source=gbs_ge_summary_r\&cad $=0 \# v=$ onepage $\& q \& f=$ false

Herrero, P.(2012). La interacción comunicativa en el proceso de enseñanza-aprendizaje. Revista de investigación y Docencia Creativa,(1),138-143.

Lacunza, A., Castro Solano, A., y Contini, N. (2009) Habilidades sociales preescolares: una escala para niños de contextos de pobreza. Revista de Psicología, XXVII (1), 4-28.

Lora, L. N. (2012). Niños y madres que permanecen en establecimientos carcelarios: escenarios de conflicto, Universidad Nacional de Mar del Plata, XII Jornadas Nacionales de Filosofía y Ciencias Políticas, Facultad de Derecho, 14-17 de noviembre 2012.

McGinnis, E., y Goldtein, A. (1990). Programa de habilidades para la infancia temprana. Recuperado de file:///C:/Users/margarita/Downloads/habilidades_infancia\%20(3).pdf

Medeiros, J. A. (2015). Mujeres privadas de libertad: análisis con perspectiva de género. El caso de una penitenciaria femenina en el Estado de Pará (Amazonia) en Brasil. Revista científica de primavera, Vol. (XIX), $\mathrm{N}^{\circ} 1$.

Niebla, E. (2014). Los derechos de niñas y niños de madres privadas de la libertad en el centro de rehabilitación social Quevedo (Tesis de pregrado). Universidad Regional Autónoma de los Andes, Ecuador.

Niño, V.M. (2011). Metodología de la Investigación: Diseño y ejecución. Recuperado de https://books.google.com.co/books?id=CyejDwAAQBAJ\&printsec=frontcover\&hl=es\&s ource=gbs_ge_summary_r\&cad=0\#v=onepage $\& \mathrm{q} \& \mathrm{f}=$ false

Peralta, J., (2000). Adquisición y desarrollo del lenguaje y la comunicación: una visión pragmática constructivista centrada en los contextos límite. Revista Interdisciplinaria de Filosofía y Psicología, (7), 54-66.

Pérez, P., y Salmerón, T. (2006). Desarrollo de la comunicación y del lenguaje: indicadores de preocupación. Pediatría Atención Primaria, VIII(32),111-125.

Puello, S. N., Silva, P. M., y Silva, A. (2014). Limites, reglas, comunicación en familia monoparental con hijos adolescentes. Interamerican Journal of Psychology, Vol. (10), $\mathrm{n}^{\circ}$ 2, 225- 246

Rizo, M. (2006). La interacción y la comunicación desde los enfoques de la psicología social y la sociología fenomenológica. Revista Análisi, 33, 45 - 62. 
Tejera,F., y Cardoso,A. (2015). Tratamiento de las habilidades comunicativas en el contexto universitario. Revista Universidad y Sociedad [seriada en línea], 7 (3). pp. 168-172.

Schmidt, V., Maglio, A., Messoulam, N., Molina, M., y González, A. (2010). La comunicación del adolescente con sus padres: construcción y validación de una escala desde un enfoque mixto. Interamerican Journal of Psychology, Vol. (44), n², 299-311.

Sousa,V.,Driessnack,M. y Costa,I. (2007). Revisión de diseños de investigación resaltantes para enfermería. parte 1: diseños de investigación cuantitativa. Revista Latino-am Enfermagem, 15(3),s.p.

Vite, E. I., y Reyes, N. L. (2016). La afectación familiar por la privación de libertad. Revista científica dominio de las ciencias, Vol. (2), 257-268. 


\section{PARA CITAR EL ARTÍCULO INDEXADO.}

Cortes Morejón, M. E., Ortiz López, N. G., \& Cabrera Martínez, L. Y. (2020). Procesos de interacción comunicacional de niños y niñas cuya madre está privada de la libertad en el Centro de Rehabilitación Social Femenino de Quito. Conciencia Digital,3(4), 102-121. https://doi.org/10.33262/concienciadigital.v3i4.1429

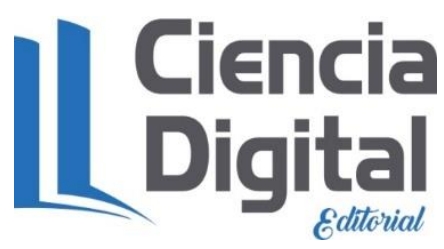

El artículo que se publica es de exclusiva responsabilidad de los autores y no necesariamente reflejan el pensamiento de la Revista Conciencia Digital.

El artículo queda en propiedad de la revista y, por tanto, su publicación parcial y/o total en otro medio tiene que ser autorizado por el director de la Revista Conciencia Digital.
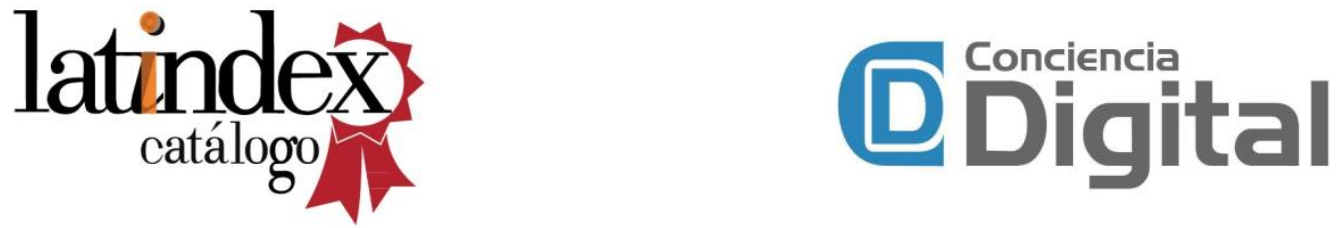